\title{
A Dual Route Model of Empathy: A Neurobiological Prospective
}

\author{
Chi-Lin Yu ${ }^{1}$ and Tai-Li Chou ${ }^{1,2 *}$ \\ ${ }^{1}$ Department of Psychology, National Taiwan University, Taipei, Taiwan, ${ }^{2}$ Center for Advanced Study in the Behavioral \\ Sciences, Stanford University, Stanford, CA, United States
}

Keywords: empathy, affective empathy, cognitive empathy, dual routes, social neuroscience

\section{INTRODUCTION}

Human beings are physically independent but are psychologically connected. We spend a lot of time dealing with others' intentions, experiences, and internal states. The term empathy describes these phenomena. Previous studies defined empathy as "the ability and tendency to share and understand others' internal state" (Zaki and Ochsner, 2012). Consistent with this definition, accumulated empirical evidence showed that empathy is a multifaceted construct composed of two components, including affective empathy and cognitive empathy (Hoffman, 1984; Decety and Jackson, 2004; Singer, 2006; Uddin et al., 2007; Shamay-Tsoory et al., 2009; Barrett et al., 2016).

Recently, ever-growing studies have considered empathy under different viewpoints (e.g., developmental trajectories, naturalism, disordered population, and so on), and have summarized the independence and non-independence of affective empathy and cognitive empathy (Barrett et al., 2016). These studies provide significant contributions to the progress of empathy research.

OPEN ACCESS

Edited by:

$\mathrm{LiHu}$,

Institute of Psychology (CAS), China

Reviewed by:

Xiaojun Cheng,

Shenzhen University, China

Qi Li,

University of Chinese Academy of

Sciences (UCAS), China

*Correspondence:

Tai-Li Chou

tlchou25@ntu.edu.tw

Specialty section:

This article was submitted to

Emotion Science,

a section of the journal

Frontiers in Psychology

Received: 07 May 2018

Accepted: 25 October 2018

Published: 13 November 2018

Citation:

Yu C-L and Chou T-L (2018) A Dual Route Model of Empathy: A Neurobiological Prospective.

Front. Psychol. 9:2212

doi: 10.3389/fpsyg.2018.02212
However, we are not yet to fully understand the neurocognitive mechanisms between the affective empathy and cognitive empathy. Three major limitations restrict our knowledge toward empathy. First, it is rare to characterize cognitive and affective empathy in terms of processing speed and involvement of consciousness. Second, previous research often studies cognitive and affective empathy separately. It is hard to identify and explain the interactive nature between these two components. Third, influential factors, including attention and prior knowledge, have not yet been considered in the framework of empathy, thus under-estimating their impacts on empathy processing. Attention and prior knowledge are closely linked with the two aforementioned limitations, specifically processing speed and consciousness involvement as well as the integration of affective empathy and cognitive empathy. Thus, these two factors are discussed in the present opinion. To address these limitations, the present opinion proposes a novel and general framework to summarize both behavioral and neural evidence in the literature. The proposed dual route model of empathy is mainly composed of an automatic, fast, and specific "lower route" with affective empathy as well as a complex, slow, and iterative "higher route" with cognitive empathy. This proposed empathy model aims to integrate these two routes and to include the influences of attention and prior knowledge.

Previously, in the domain of emotion neuroscience, Joseph LeDuox, a pioneer to study emotions, proposed a framework with two parallel neural systems, namely a low road and a high road (LeDoux, 1998). Specifically, the low road, which is a fast, subcortical, short-latency pathway with minimal cortical involvement, directly conveys sensory information from the thalamus to the amygdala, allowing stimuli to be processed automatically without consciousness and awareness (Davis, 1992; LeDoux, 1995, 1997). These features of rapid and automatic processes construct the low road emotion processes. In contrast, the high road indicates the visual pathways from retina neuron to visual cortex, and then connects to inferior temporal lobe for processing higher level consciousness of emotion feeling, and finally directs to the amygdala (LeDoux, 1998). These features of slow processing and more involvement of consciousness establish the high road of emotion. 
The present opinion hypothesizes that empathy also has a similar dual route system, which includes an automatic, fast and lower-level route (i.e., lower route) and a complex, slow and higher-level route (i.e., higher route), inside our brain (Figure 1). The rest of the paper is organized as a series of introductions for each component of empathy. Also, we consider these components in the proposed dual route model of empathy in order to obtain the whole picture of the empathy processing.

\section{LOWER ROUTE: AFFECTIVE EMPATHY}

First of all, the lower route is an efficient, automatic and fast process with minimal involvement of consciousness. Affective empathy, which defined as one's emotional, sensorimotor, and visceral response to the affective state of other, encompasses the mentioned-above efficient, automatic and fast features of the lower route. Affective empathy is also usually described by different but aligned terms like "experience sharing" (Zaki and Ochsner, 2012; Barrett et al., 2016) or “empathic concern” (Davis, 1994).

From the perspective of behavioral studies, affective empathy captures the phenomena that people automatically share the experiences with the targets they observe, such as arousal (Vaughan and Lanzetta, 1980; Levenson and Ruef, 1992), moods (Neumann and Strack, 2000) and facial expression (Dimberg and Thunberg, 1998). It has long been shown that affective empathy can rapidly occur (Dimberg and Thunberg, 1998), even outside of our consciousness and awareness (Neumann and Strack, 2000). It is also suggested that affective empathy quickly emerges and appears stable in early development (Knafo et al., 2008; RothHanania et al., 2011; Davidov et al., 2013). For example, infants express emotional feeling while hearing the sound of other's cries rather than hearing their own cries (Sagi and Hoffman, 1976; Martin and Clark, 1982) and are able to respond to other's facial expressions in the first few weeks of life (Haviland and Lelwica, 1987). Overall, behavioral findings support the idea that affective empathy is a basic and primitive beginning of empathy (Hoffman, 2001).

From the viewpoints of neural evidence, research studying affective empathy over two decades consistently reveal a specific system, the mirror neuron system, as the underpinning of experience sharing processes (Di Pellegrino et al., 1992; Iacoboni, 2009; Rizzolatti and Sinigaglia, 2010, 2016). The mirror neuron system is thought to comprise the inferior frontal cortex, the premotor areas, and the insula. When we observe that others are experiencing some sensorimotor or affective information, our mirror neuron system provides a simple mechanism to generate the representations of other's states by simulating neuronal activities similar to the observed ones, thus allowing an automatic share of others' experiences (Iacoboni et al., 2005; Heyes, 2011). Furthermore, regions in the mirror neuron system demonstrate automatic (Iacoboni et al., 2005; Heyes, 2011) and unconscious information transition (Carr et al., 2003; Leslie et al., 2004). Moreover, the neural profiles of affective empathy are specifically located in the mirror neuron system with little requirements of additional cortical regions.
Supported by both behavioral and neural evidence, affective empathy, which includes basic, unconscious, automatic and fast processes, constructs the lower route in the model.

\section{HIGHER ROUTE: COGNITIVE EMPATHY}

The second route in the model is the higher route, which is a slow and complex process with efforts, consciousness, and elaborated neural profiles. Cognitive empathy, which refers to the ability to understand or explicitly reason the subjective mental states, perspectives or intentions of others (Gopnik and Wellman, 1992), establishes the higher route of the model. Cognitive empathy is also known as "mentalizing" (Barrett et al., 2016), "Theory of Mind" (Premack and Woodruff, 1978) or "perspective taking" (Davis, 1994).

From the perspective of behavioral findings, several demonstrations suggest that cognitive empathy is effortful (Lin et al., 2010) and requires attention and time (Gilovich et al., 2000; Keysar et al., 2000). People would make wrong inferences on the internal states of others because of the attentional disruption or limited time. In addition, developmental findings indicate that cognitive empathy, which relies on the involvement of other cognitive abilities (e.g., inhibition, execute functions), does not appear until the first year (Onishi and Baillargeon, 2005). Also, an anchoring and adjustment mechanism of cognitive empathy is proposed to describe its complex processing (Gilovich et al., 2000; Barrett et al., 2016). Namely, people first use their awareness and effort to establish personal theories or assumptions about others' psychological states, and then further adjust and correct the generated theories during interactions. In brief, all the arguments provided by the behavioral literature suggest a more elaborated, complex, and high-level processing of cognitive empathy (Carlson and Moses, 2001; Wellman et al., 2001).

From the viewpoints of neuroscience research, imaging studies also reveal a complete neural profile for cognitive empathy. Engaged brain regions mainly include dorsal, middle, and ventral medial prefrontal cortex (DMPFC, MMPFC, VMPFC), precuneus (PC), and temporoparietal junction (TPJ). These areas are selectively activated when subjects make inferences on the information about others' mental states. The impairments of cognitive empathy are also highly correlated with atypical activity of these regions. For example, individuals with autism spectrum disorders (ASD), who have difficulties in cognitive empathy, often exhibit altered brain patterns in cognitive empathy-associated regions in rest (Picci et al., 2016; Hull et al., 2017) or under cognitive processing (Philip et al., 2012; Maximo et al., 2014; Picci et al., 2016). Rather than depending on a single system as shown in the lower route, cognitive empathy requires the involvements of a variety of brain regions (i.e., TPJ, PC, VMPFC, MMPFC, and DMPFC). These regions also demonstrate a complex hierarchical structure (Van Overwalle and Vandekerckhove, 2013). That is, the TPJ processes the inferences of others' intentions and belief, the MPFC associates with the inferences of others' traits or stable characteristics, and the iterative re-processing between 


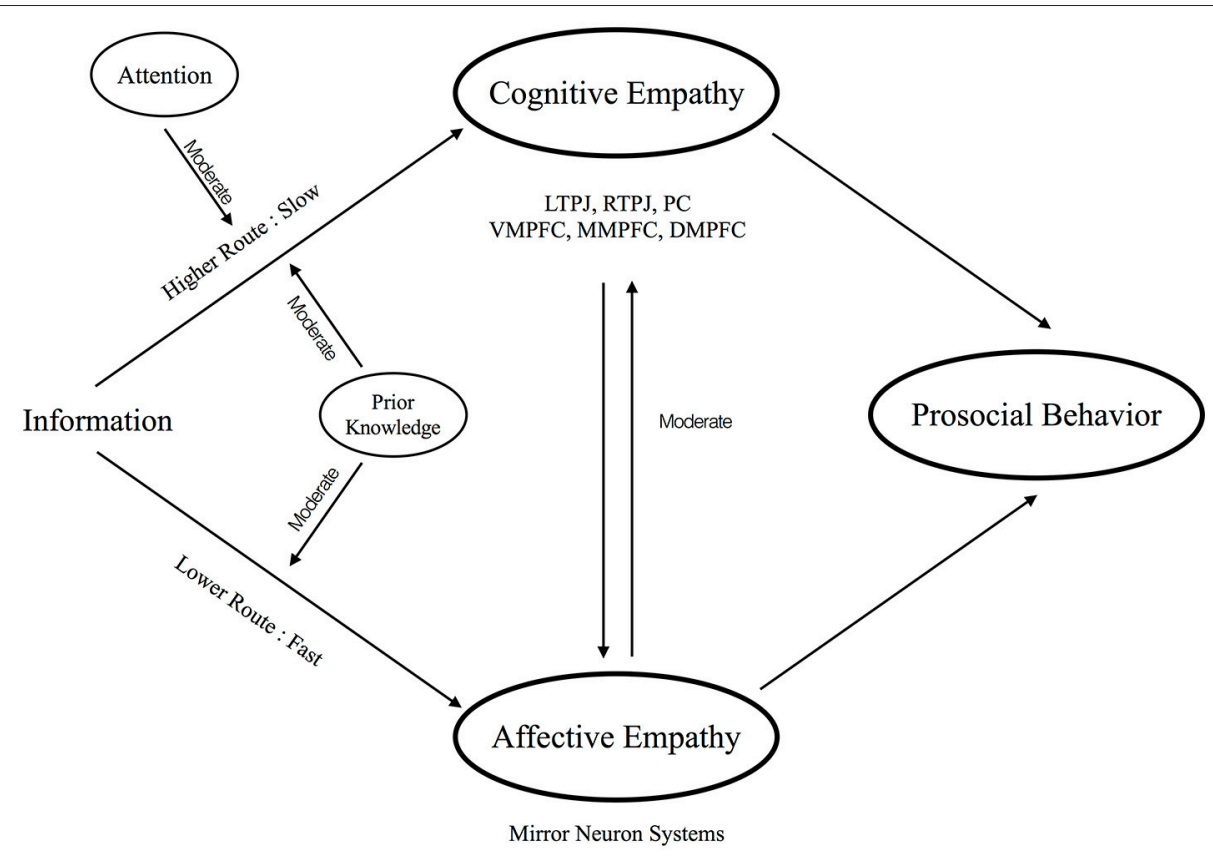

FIGURE 1 | The conceptual visualization for the proposed dual route model of empathy.

TPJ and MPFC further extracts the information of others' mental states (Van Overwalle and Baetens, 2009; Van Overwalle and Vandekerckhove, 2013). This slow, complex, and iterative processing echoes the aforementioned anchoring and adjustment mechanism.

Moreover, we hypothesize attention to moderate the higher route. As mentioned, participants could not correctly infer others' belief or knowledge when distracted (Gilovich et al., 2000). Further studies even argued that people appear to become "mindblind," meaning that they fail to mentalize others when they have no sufficient attention resource (Lin et al., 2010). Together, these findings not only state the effortful and complex process in the higher route, but also highlight the moderating role of attention on the higher route.

Evidenced by arguments from behavioral and neural findings, cognitive empathy with slow, complex and higher-level features is the essence of the higher route in the empathy framework.

\section{HYPOTHESIZED CONNECTIONS}

In addition to the aforementioned two routes constructing the main structure of the dual route model of empathy, several hypothesized connections are proposed to elaborate the relationships between two routes.

First, the connections between affective and cognitive empathy may have influences on each other, thereby impacting the level of empathy processing. On one hand, there may be a modulation from the lower route to higher route. For example, our ability to mentalize others depends on whether we have shared their feelings. On the other hand, a modulation from the higher route to lower route may exist. For instance, when we fully know about others' mind, we probably can be more able to know others' feeling. Neural evidence echoes this speculation by suggesting the influences of connectivity between regions of affective and cognitive empathy on social abilities (Fishman et al., 2014; Libero et al., 2014). Also, a behavioral study with ASD supported this argument by suggesting that affective empathy and cognitive empathy are inter-dependent (Bos and Stokes, 2018). Whilst it is a topic under investigations, the present opinion highlights an interactive connection between two routes.

Second, our prior knowledge, such as impression and familiarity about others, may affect the processes in the lower and higher route (Han and Northoff, 2008; Serino et al., 2009; Xu et al., 2009; Liew et al., 2011). For example, previous evidence showed that both cognitive and affective empathy-associated brain regions are more activated when people observe familiar friends than unfamiliar strangers in pain (Meyer et al., 2012). A number of studies further suggested that the brain regions for impression formation are highly overlapped with the areas supporting cognitive empathy (Mitchell et al., 2004; Schiller et al., 2009; Yu et al., 2016). According to these lines of evidence, the present opinion suggests that our prior knowledge constructs a moderated role on both the lower and higher routes.

Third, prosocial behavior is the output that flows from the two routes. That is, people who share and understand others' mind will finally care about others and generate desires to help others (Barrett et al., 2016). Supporting evidence connects both affective and cognitive empathy with prosocial behavior (Snyder and Lopez, 2009). For example, brain regions of affective empathy (Singer et al., 2008; Hein et al., 2010; Masten et al., 2011) and cognitive empathy (Rameson et al., 2012; Waytz et al., 2012) 
can both predict willingness to perform prosocial behaviors. Furthermore, affective empathy and cognitive empathy may have different effects on prosocial behavior. On the one hand, since affective empathy is a fast and automatic route without involvements of consciousness, it is most often elicited by explicit cues such as facial expression. In consequence, the processes of affective empathy toward prosocial behavioral can be skewed by the accessibility of the target. When a person can directly assess the cues from others, he/she can elicit more affective empathy and produce more prosocial behavioral. On the other hand, because the involvement of consciousness, cognitive empathy can support prosocial behavior but not always do so. For example, people may not show prosocial behavior to their enemies, although people can still mentalize them. In brief, integration of these two routes can affect prosocial behaviors, and is by no means monotonic.

In short, although few studies shed light on these connections, the present opinion considers them in the model as influential factors underlying the complex processes of empathy.

\section{REFERENCES}

Barrett, L. F., Lewis, M., and Haviland-Jones, J. M. (2016). Handbook of Emotions. New York, NY: Guilford Publications.

Bos, J., and Stokes, M. A. (2018). Cognitive empathy moderates the relationship between affective empathy and wellbeing in adolescents with autism spectrum disorder. Euro. J. Develop. Psychol. 1-14. doi: 10.1080/17405629.2018.1444987

Carlson, S. M., and Moses, L. J. (2001). Individual differences in inhibitory control and children's theory of mind. Child Develop. 72, 1032-1053. doi: $10.1111 / 1467-8624.00333$

Carr, L., Iacoboni, M., Dubeau, M. C., Mazziotta, J. C., and Lenzi, G. L. (2003). Neural mechanisms of empathy in humans: a relay from neural systems for imitation to limbic areas. Proc. Natl. Acad. Sci. U.S.A. 100, 5497-5502. doi: 10.1073/pnas.0935845100

Davidov, M., Zahn-Waxler, C., Roth-Hanania, R., and Knafo, A. (2013). Concern for others in the first year of life: Theory, evidence, and avenues for research. Child Develop. Perspect. 7, 126-131. doi: 10.1111/cdep.12028

Davis, M. (1992). The role of the amygdala in fear and anxiety. Ann. Rev. Neurosci. 15, 353-375. doi: 10.1146/annurev.ne.15.030192.002033

Davis, M. H. (1994). Empathy: A Social Psychological Approach. Boulder, CO: Westview Press.

Decety, J., and Jackson, P. L. (2004). The functional architecture of human empathy. Behav. Cogn. Neurosci. Rev. 3, 71-100. doi: $10.1177 / 1534582304267187$

Di Pellegrino, G., Fadiga, L., Fogassi, L., Gallese, V., and Rizzolatti, G. (1992). Understanding motor events: a neurophysiological study. Exper. Brain Res. 91, 176-180. doi: 10.1007/BF00230027

Dimberg, U., and Thunberg, M. (1998). Rapid facial reactions to emotional facial expressions. Scand. J. Psychol. 39, 39-45. doi: 10.1111/1467-9450.00054

Fishman, I., Keown, C. L., Lincoln, A. J., Pineda, J. A., and Müller, R. A. (2014). Atypical cross talk between mentalizing and mirror neuron networks in autism spectrum disorder. JAMA Psychiatry 71, 751-760. doi: 10.1001/jamapsychiatry.2014.83

Gilovich, T., Medvec, V. H., and Savitsky, K. (2000). The spotlight effect in social judgment: an egocentric bias in estimates of the salience of one's own actions and appearance. J. Person. Soc. Psychol. 78:211. doi: 10.1037/0022-3514.78.2.211

Gopnik, A., and Wellman, H. M. (1992). Why the child's theory of mind really is a theory. Mind Lang. 7, 145-171. doi: 10.1111/j.1468-0017.1992.tb 00202.x

Han, S., and Northoff, G. (2008). Culture-sensitive neural substrates of human cognition: a transcultural neuroimaging approach. Nat. Rev. Neurosci. 9:646. doi: $10.1038 / \mathrm{nrn} 2456$

\section{CONCLUSIONS}

The relationship between the higher route and lower route is closely connected. By integrating both behavioral and neural evidence, the present opinion proposes a general framework, the dual route model, as a mechanism to explain the underlying process of empathy.

\section{AUTHOR CONTRIBUTIONS}

All authors listed have made a substantial, direct and intellectual contribution to the work, and approved it for publication.

\section{FUNDING}

This research was supported by grants from the Ministry of Science and Technology of Taiwan (MOST 105-2410-H-002053, 106-2410-H-002-079) and Science and Technology Policy Research and Information Center of NARLabs to T-LC.

Haviland, J. M., and Lelwica, M. (1987). The induced affect response: 10-weekold infants' responses to three emotion expressions. Develop. Psychol. 23:97. doi: 10.1037/0012-1649.23.1.97

Hein, G., Silani, G., Preuschoff, K., Batson, C. D., and Singer, T. (2010). Neural responses to ingroup and outgroup members' suffering predict individual differences in costly helping. Neuron 68, 149-160. doi: 10.1016/j.neuron.2010.09.003

Heyes, C. (2011). Automatic imitation. Psychol. Bull. 137:463. doi: $10.1037 / \mathrm{a} 0022288$

Hoffman, M. L. (1984). Hoffman, M. L. (1984). "Interaction of affect and cognition on empathy," in Emotions, Cognition, and Behavior, eds C. E. Izard, J. Kagan, and R. B. Zajonc (New York, NY: Cambridge University Press), 103-131.

Hoffman, M. L. (2001). Empathy and Moral Development: Implications for Caring and Justice. Cambridge: Cambridge University Press.

Hull, J. V., Jacokes, Z. J., Torgerson, C. M., Irimia, A., and Van Horn, J. D. (2017). Resting-state functional connectivity in autism spectrum disorders: a review. Front. Psychiatry 7:205. doi: 10.3389/fpsyt.2016.00205

Iacoboni, M. (2009). Imitation, empathy, and mirror neurons. Ann. Rev. Psychol. 60, 653-670. doi: 10.1146/annurev.psych.60.110707.163604

Iacoboni, M., Molnar-Szakacs, I., Gallese, V., Buccino, G., Mazziotta, J. C., and Rizzolatti, G. (2005). Grasping the intentions of others with one's own mirror neuron system. PLoS Biol. 3:e79. doi: 10.1371/journal.pbio.0030079

Keysar, B., Barr, D. J., Balin, J. A., and Brauner, J. S. (2000). Taking perspective in conversation: the role of mutual knowledge in comprehension. Psychol. Sci. 11, 32-38. doi: 10.1111/1467-9280.00211

Knafo, A., Zahn-Waxler, C., Van Hulle, C., Robinson, J. L., and Rhee, S. H. (2008). The developmental origins of a disposition toward empathy: genetic and environmental contributions. Emotion 8:737. doi: 10.1037/a0014179

LeDoux, J. (1998). The Emotional Brain: The Mysterious Underpinnings of Emotional Life. New York, NY: Simon and Schuster.

LeDoux, J. E. (1995). Emotion: clues from the brain. Annu. Rev. Psychol. 46, 209-235. doi: 10.1146/annurev.ps.46.020195.001233

LeDoux, J. E. (1997). Emotion, memory and the brain. Sci. Am. 7, 68-75.

Leslie, K. R., Johnson-Frey, S. H., and Grafton, S. T. (2004). Functional imaging of face and hand imitation: towards a motor theory of empathy. Neuroimage 21, 601-607. doi: 10.1016/j.neuroimage.2003.09.038

Levenson, R. W., and Ruef, A. M. (1992). Empathy: a physiological substrate. J. Person. Soc. Psychol. 63:234. doi: 10.1037/0022-3514.63.2.234

Libero, L. E., Maximo, J. O., Deshpande, H. D., Klinger, L. G., Klinger, M. R., and Kana, R. K. (2014). The role of mirroring and mentalizing networks in mediating action intentions in autism. Mol. Autism 5:50. doi: $10.1186 / 2040-2392-5-50$ 
Liew, S. L., Han, S., and Aziz-Zadeh, L. (2011). Familiarity modulates mirror neuron and mentalizing regions during intention understanding. Hum. Brain Mapp. 32, 1986-1997. doi: 10.1002/hbm.21164

Lin, S., Keysar, B., and Epley, N. (2010). Reflexively mindblind: using theory of mind to interpret behavior requires effortful attention. J. Exper. Soc. Psychol. 46, 551-556. doi: 10.1016/j.jesp.2009.12.019

Martin, G. B., and Clark, R. D. (1982). Distress crying in neonates: species and peer specificity. Develop. Psychol. 18:3. doi: 10.1037/0012-1649.18.1.3

Masten, C. L., Morelli, S. A., and Eisenberger, N. I. (2011). An fMRI investigation of empathy for 'social pain'and subsequent prosocial behavior. Neuroimage 55, 381-388. doi: 10.1016/j.neuroimage.2010.11.060

Maximo, J. O., Cadena, E. J., and Kana, R. K. (2014). The implications of brain connectivity in the neuropsychology of autism. Neuropsychol. Rev. 24, 16-31. doi: 10.1007/s11065-014-9250-0

Meyer, M. L., Masten, C. L., Ma, Y., Wang, C., Shi, Z., Eisenberger, N. I., et al. (2012). Empathy for the social suffering of friends and strangers recruits distinct patterns of brain activation. Soc. Cogn. Affect. Neurosci. 8, 446-454. doi: 10.1093/scan/nss019

Mitchell, J. P., Macrae, C. N., and Banaji, M. R. (2004). Encoding-specific effects of social cognition on the neural correlates of subsequent memory. J. Neurosci. 24, 4912-4917. doi: 10.1523/JNEUROSCI.0481-04.2004

Neumann, R., and Strack, F. (2000). "Mood contagion": the automatic transfer of mood between persons. J. Person. Soc. Psychol. 79:211. doi: 10.1037/0022-3514.79.2.211

Onishi, K. H., and Baillargeon, R. (2005). Do 15-month-old infants understand false beliefs? Science 308, 255-258. doi: 10.1126/science.1107621

Philip, R. C., Dauvermann, M. R., Whalley, H. C., Baynham, K., Lawrie, S. M., and Stanfield, A. C. (2012). A systematic review and meta-analysis of the fMRI investigation of autism spectrum disorders. Neurosci. Biobehav. Rev. 36, 901-942. doi: 10.1016/j.neubiorev.2011.10.008

Picci, G., Gotts, S. J., and Scherf, K. S. (2016). A theoretical rut: revisiting and critically evaluating the generalized under/over-connectivity hypothesis of autism. Develop. Sci. 19, 524-549. doi: 10.1111/desc.12467

Premack, D., and Woodruff, G. (1978). Does the chimpanzee have a theory of mind? Behav. Brain Sci. 1, 515-526. doi: 10.1017/S0140525X00076512

Rameson, L. T., Morelli, S. A., and Lieberman, M. D. (2012). The neural correlates of empathy: experience, automaticity, and prosocial behavior. J. Cogn. Neurosci. 24, 235-245. doi: 10.1162/jocn_a_00130

Rizzolatti, G., and Sinigaglia, C. (2010). The functional role of the parieto-frontal mirror circuit: interpretations and misinterpretations. Nat. Rev. Neurosci. 11:264. doi: 10.1038/nrn2805

Rizzolatti, G., and Sinigaglia, C. (2016). The mirror mechanism: a basic principle of brain function. Nat. Rev. Neurosci. 17:757. doi: 10.1038/nrn.2016.135

Roth-Hanania, R., Davidov, M., and Zahn-Waxler, C. (2011). Empathy development from 8 to 16 months: early signs of concern for others. Infant Behav. Develop. 34, 447-458. doi: 10.1016/j.infbeh.2011.04.007

Sagi, A., and Hoffman, M. L. (1976). Empathic distress in the newborn. Develop. Psychol. 12:175. doi: 10.1037/0012-1649.12.2.175

Schiller, D., Freeman, J. B., Mitchell, J. P., Uleman, J. S., and Phelps, E. A. (2009). A neural mechanism of first impressions. Nat. Neurosci. 12:508. doi: $10.1038 / \mathrm{nn} .2278$

Serino, A., Giovagnoli, G., and Làdavas, E. (2009). I feel what you feel if you are similar to me. PLoS ONE 4:e4930. doi: 10.1371/journal.pone.0004930
Shamay-Tsoory, S. G., Aharon-Peretz, J., and Perry, D. (2009). Two systems for empathy: a double dissociation between emotional and cognitive empathy in inferior frontal gyrus versus ventromedial prefrontal lesions. Brain 132, 617-627. doi: 10.1093/brain/awn279

Singer, T. (2006). The neuronal basis and ontogeny of empathy and mind reading: review of literature and implications for future research. Neurosci. Biobehav. Rev. 30, 855-863. doi: 10.1016/j.neubiorev.2006.06.011

Singer, T., Snozzi, R., Bird, G., Petrovic, P., Silani, G., Heinrichs, M., et al. (2008). Effects of oxytocin and prosocial behavior on brain responses to direct and vicariously experienced pain. Emotion 8:781. doi: 10.1037/a0014195

Snyder, C. R., and Lopez, S. J. (2009). Oxford Handbook of Positive Psychology. New York, NY: Oxford University Press.

Uddin, L. Q., Iacoboni, M., Lange, C., and Keenan, J. P. (2007). The self and social cognition: the role of cortical midline structures and mirror neurons. Trends Cogn. Sci. 11, 153-157. doi: 10.1016/j.tics.2007.01.001

Van Overwalle, F., and Baetens, K. (2009). Understanding others' actions and goals by mirror and mentalizing systems: a meta-analysis. Neuroimage 48, 564-584. doi: 10.1016/j.neuroimage.2009.06.009

Van Overwalle, F., and Vandekerckhove, M. (2013). Implicit and explicit social mentalizing: dual processes driven by a shared neural network. Front. Hum. Neurosci. 7:560. doi: 10.3389/fnhum.2013.00560

Vaughan, K. B., and Lanzetta, J. T. (1980). Vicarious instigation and conditioning of facial expressive and autonomic responses to a model's expressive display of pain. J. Person. Soc. Psychol. 38:909. doi: 10.1037/0022-3514.38.6.909

Waytz, A., Zaki, J., and Mitchell, J. P. (2012). Response of dorsomedial prefrontal cortex predicts altruistic behavior. J. Neurosci. 32, 7646-7650. doi: 10.1523/JNEUROSCI.6193-11.2012

Wellman, H. M., Cross, D., and Watson, J. (2001). Meta-analysis of theory-ofmind development: the truth about false belief. Child Develop. 72, 655-684. doi: 10.1111/1467-8624.00304

Xu, X., Zuo, X., Wang, X., and Han, S. (2009). Do you feel my pain? Racial group membership modulates empathic neural responses. J. Neurosci. 29, 8525-8529. doi: 10.1523/JNEUROSCI.2418-09.2009

$\mathrm{Yu}$, C. L., Wang, M. Y., and Hu, J. F. (2016). Valence processing of first impressions in the dorsomedial prefrontal cortex: a near-infrared spectroscopy study. Neuroreport 27, 574-579. doi: 10.1097/WNR.0000000000 000571

Zaki, J., and Ochsner, K. N. (2012). The neuroscience of empathy: progress, pitfalls and promise. Nat. Neurosci. 15:675. doi: 10.1038/ nn.3085

Conflict of Interest Statement: The authors declare that the research was conducted in the absence of any commercial or financial relationships that could be construed as a potential conflict of interest.

The reviewer, QL, and handling Editor declared their shared affiliation.

Copyright (C) $2018 \mathrm{Yu}$ and Chou. This is an open-access article distributed under the terms of the Creative Commons Attribution License (CC BY). The use, distribution or reproduction in other forums is permitted, provided the original author(s) and the copyright owner(s) are credited and that the original publication in this journal is cited, in accordance with accepted academic practice. No use, distribution or reproduction is permitted which does not comply with these terms. 